\title{
Lutschtabletten gegen Mundtrockenheit
}

Mundtrockenheit wird nicht nur als unangenehm empfunden, sondern kann bei unzureichender Mundpflege orale Entzündungen und kariöse Läsionen begünstigen. Daher ist es wichtig Mundtrockenheit vorzubeugen und die Mundschleimhaut im Akutfall zu schützen und aktiv zu pflegen. Die miradent $A Q U A M E D \&$ MundtrockenheitsLutschtabletten fördern den Speichelfluss, entfalten ein angenehmes, feuchtes Mundgefühl und helfen so dabei, das Mundmilieu in einem guten Zustand zu halten. Neben dem körpereigenen Enzym Lysozym enthält die Rezeptur der Lutschtablette zusätzlich zahnpflegendes Xylitol und Kalziumlaktat, das die Remineralisierung des Zahnschmelzes begünstigt. Die Tablette kann je nach Bedarf mehrmals täglich angewendet werden, die empfohlene Tagesdosis liegt bei 4 Stück. Sie enthalten weder Zucker, noch Aspartam und sind frei von Gluten.

Nach einer Pressemitteilung der Hager \& Werken GmbH \& Co. KG, Duisburg www.hagerwerken.de 